\title{
Valoración de parámetros químicos del agua en pozos de la UNAH durante 2016
}

\author{
Alexander Reyes ${ }^{1}$ \\ Marcela Godoy, David Pineda ${ }^{2}$
}

\section{RESUMEN}

El presente proyecto de investigación está orientado a hacer una valoración de los parámetros químicos del agua de pozos empleada en Ciudad Universitaria, Tegucigalpa, Honduras, durante un periodo comprendido entre 2015-2016. El objetivo primordial es determinar si el agua cumple al $100 \%$ con los requisitos mínimos de calidad para su uso humano. En caso de no cumplir con esta norma, se buscó identificar alteraciones en sus valores definidos como normales y evaluar su posible impacto en la salud de la comunidad. El muestreo se llevó a cabo en dos momentos del año: temporada seca y temporada con lluvias. La metodología y la parte analítica fueron llevadas a cabo por el laboratorio certificado del Servicio Nacional Autónomo de Acueductos y Alcantarillados (SANAA). Los resultados arrojaron datos de alteración principalmente en valores considerados secundarios en normas de calidad del agua. Los datos fuera de los parámetros normales son: Conductividad eléctrica, dureza total, Sulfatos, Nitrógeno amoniacal y el Hierro. Los efectos de una alta concentración salina y de sulfatos no están bien marcados por la guía de calidad de la OMS (2006) sin embargo, se han reportados algunos efectos nocivos sobre la salud de las personas con respecto a un alto contenido de sulfatos que se relaciona con un efecto laxante. Otro efecto dañino del alto contenido de sales estaría relacionado con la pobre calidad del agua en sus datos organolépticos y posibles daños en las tuberías del sistema de abastecimiento del campus.

Palabras clave: aguas subterráneas, pozo, contaminación, calidad, parámetros químicos.

${ }^{1}$ Beneficiario de una beca básica de la DICYP. Profesor de la Facultad de Ciencias Químicas y Farmacia, UNAH: alexanderadra@gmail.com

2 Beneficiarios de una beca básica de la DICYP. Profesores del Departamento de Ingeniería Química, Facultad de Ingeniería, UNAH: marcelamgz@gmail.com 


\section{RESUMEN}

The present research project is focused on making an assessment of chemistry profile of water from well. This is used in Ciudad Universitaria, Tegucigalpa, Honduras between a time period from 2015-2016. The main objective of this research is to determine if the water used by that community meets the parameters at 100 percent to be considered and used by human activities. In case of parameters did not meet the limits, the goal was to identify altered values and to evaluate a possible impact of them in people's health into the campus community. The sampling was developed in two specific periods of time: dry season and rainy season. The methods and Analytical chemistry was conducted by the certified lab of Servicio Nacional Autónomo de Acueductos y Alcantarillados SANAA. The results revealed some data altered but mainly on parameters considered as secondary on quality standards of water. Those parameters were; electric conductivity, total hardness, sulfates, nitrogen from ammonia, and iron. The effects resulting of high concentrations of salts are not considered harmful for human health and not well-defined by WHO (world Health Organization, 2006). However, some laxative effects have been reported. Other noxious effects reported by high concentrations of salts might be related to poor quality of fresh water on its physic characteristics as well as some possible damage to the pipe lines systems into the campus.

Keywords: ground waters, well, pollution, quality, chemical parameters. 


\section{INTRODUCCIÓN}

El proyecto tuvo como objetivo primordial llevar a cabo un análisis de aguas subterráneas 0 agua de pozos que abastecen las instalaciones de la comunidad universitaria para determinar si el agua que se usa para actividades variadas, dentro de la comunidad universitaria cumple con la norma técnica específica para agua potable que establecen los libros oficiales. Se llevó a cabo una valoración del perfil químico del agua que se utiliza por los miembros de la ciudad universitaria y demás miembros de la comunidad local durante el periodo 2015 - 2016. Tomando en cuenta que se ha reportado la presencia de color $u$ olor en el agua y altas concentraciones de sales, las cuales provocan duda en el usuario de su completa inocuidad. La investigación consideró los estudios que ha llevado a cabo SERNA a través de CESCCO la temática se ha trabajado desde 1969, además de los estudios que ha llevado a cabo el SANAA en los cuales se ha identificado problemas con algunos parámetros fisicoquímicos en el agua.

El propósito de este estudio fue evaluar el riesgo potencial que representaría a las personas de la Ciudad Universitaria una alteración de los parámetros que definen el uso seguro del agua subterránea para la salud de las personas. Este estudio fue diseñado para detectar alteraciones en los parámetros químicos, del agua que se utiliza diariamente en el campus universitario. Esto permite identificar potenciales problemas de salud derivados de la presencia de parámetros químicos en el agua que no cumplan con las normas de calidad que garanticen a la comunidad universitaria su uso seguro. Así mismo, el estudio podría implementarse también en otros centros regionales de la UNAH que se abastecen de aguas subterráneas. En caso de encontrar alteraciones y riesgos en sus parámetros químicos, un estudio pertinente ayudaría directamente a prevenir las consecuencias de un problema sanitario que afectaría un gran número de personas que además de ser perjudicadas directamente representarían un problema a la economía del sistema de salud pública del país. En términos generales, este estudio buscó identificar riesgos derivados de parámetros químicos inapropiados en el agua de pozo, prevenir cualquier potencial riesgo a la salud de los miembros de la comunidad universitaria e identificar potenciales fuentes de contaminación de los acuíferos del campus universitario en Tegucigalpa.

\section{METODOLOGÍA}

La investigación tuvo un alcance descriptivo. El objetivo primario de la investigación fué describir los parámetros químicos del agua de pozo empleada en la UNAH durante 2016. El enfoque cuantitativo precedió el estudio ya que se midieron parámetros 
químicos. A través de un muestro no probabilístico, se procedió a la toma de muestras en los pozos de Ciudad Universitaria.

Las muestras fueron tomadas en dos épocas del año distintas. La primera fue tomada en el mes de enero, época seca del año, y la segunda fue tomada en agosto, época de invierno en Tegucigalpa. En el primer muestreo las muestras fueron tomadas en 3 intervalos de tiempo distintos con el objetivo de ver variaciones durante el día. Se midió el pH en el sitio de la toma de muestra y en el laboratorio, por medio de un pHmetro. Además, se midió la temperatura con un termómetro calibrado en el sitio de muestreo. Las muestras fueron tomadas en recipientes previamente esterilizados; luego se almacenaron las muestras en hieleras para garantizar una temperatura inferior a $60 \mathrm{C}$ y fueron transportadas inmediatamente al laboratorio para posterior análisis. Los siguientes parámetros fueron determinados en el laboratorio: conductividad por medio de un conductímetro para medir la conductancia específica o resistencia eléctrica. Todos los aparatos empleados en el análisis fueron calibrados previo a ser empleados en la medición de los parámetros citados. La dureza total mediante un análisis volumétrico con EDTA (Ácido etilen di-amino tetra acético) se coloca una alícuota de agua en un matraz Erlen Meyer con indicador (Negro de Eriocromo $T$ (NET)), y luego se procede a la valoración complejométrica con la ayuda de una buerta graduada y calibrada clase A, que mide el volumen que entrega, hasta llegar al punto final. El volumen consumido se usa para determinar el número de equivalentes-gramo de EDTA que forman un complejo con los iones $\mathrm{Ca} 2+$ y Mg2+ y de esta manera de forma directa se determina los $\mathrm{mg} / \mathrm{L}$ de estos iones que son responsables de la dureza total en el agua. Hierro y Manganeso fueron determinados por medio de la técnica analítica Espectroscopia de Absorción Atómica, esta técnica se basa en la absorción de radiación óptica (luz) por parte de los atomos liberados (Hierro y Manganeso) que fueron sometidos a altas temperaturas para llevarlos a estado elemental.

La absorción de radiación permite la determinación cuantitativa de los elementos químicos citados en $\mathrm{mg} / \mathrm{L}$ o unidades equivalentes. El nitrógeno amoniacal fue determinado por Medio de la técnica analítica denominada colorimetría. Esta técnica emplea el uso de 1,10-Fenantrolina (Orto-Fenantrolina) para formar un complejo colorido que pueda absorber radiación en la región visible del espectro electromagnético. La determinación cuantitativa de la radiación absorbida es proporcional a la concentración del analito. Los Sulfatos fueron determinados por métodos turbidimétricos, con el empleo de un turbidímetro y métodos espectroscópicos, la cantidad de sólidos o sustancias salinas en suspensión incremental la dispersión de la radiación electromagnética que penetra el medio acuoso, esta perturbación es medida por un aparato que detecta la turbidez del agua basado en la cantidad de sólidos en suspen- 
sión y da lecturas en unidades NTU que son indicativo directo del material suspendido en el agua analizada.

Para el análisis estadístico y elaboración de los gráficos mostrados se empleó Microsoft Excel 365. Los gráficos permitieron determinar qué tan altos eran los valores obtenidos durante las mediciones con respecto a los valores de referencia.

\section{RESULTADOS}

Los resultados de análisis que se presentan a continuación corresponden únicamente a los parámetros químicos alterados que se encontraron después de analizar las muestras de agua de pozo tanto en temporada seca como en temporada de lluvia. Los demás parámetros en consideración como ser pH, temperatura, presencia de fosfatos, flúor, nitratos, nitritos, oxígeno disuelto y solido totales disueltos. Todos ellos tuvieron valores medidos dentro de los límites normales establecidos.

Los resultados más elevados con respecto a la conductividad se pueden apreciar en el grafico 1 y 2 , con datos hasta 6 veces más alto de los parámetros establecidos como normales tanto en enero (temporada seca) como en agosto (temporada lluviosa).

Gráfico 1. Conductividad en Pozo UNAH “Canchas de Fútbol \#4-5.

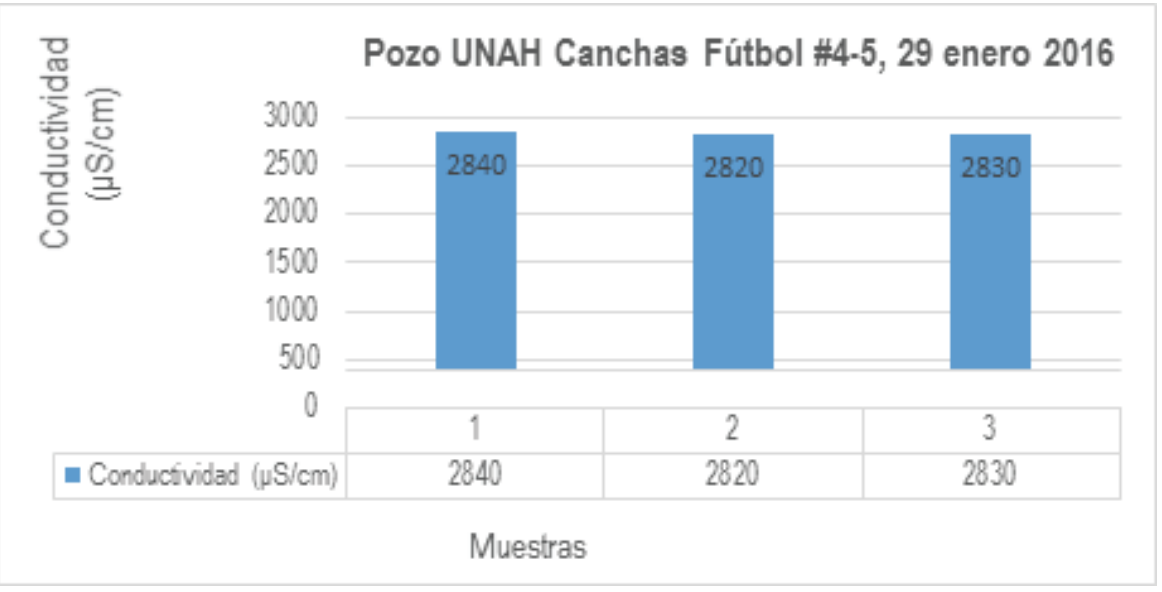

Fuente: Elaboración propia. 
Gráfico 2. Conductividad, agosto. Límite de $400 \mu \mathrm{s} / \mathrm{cm}$

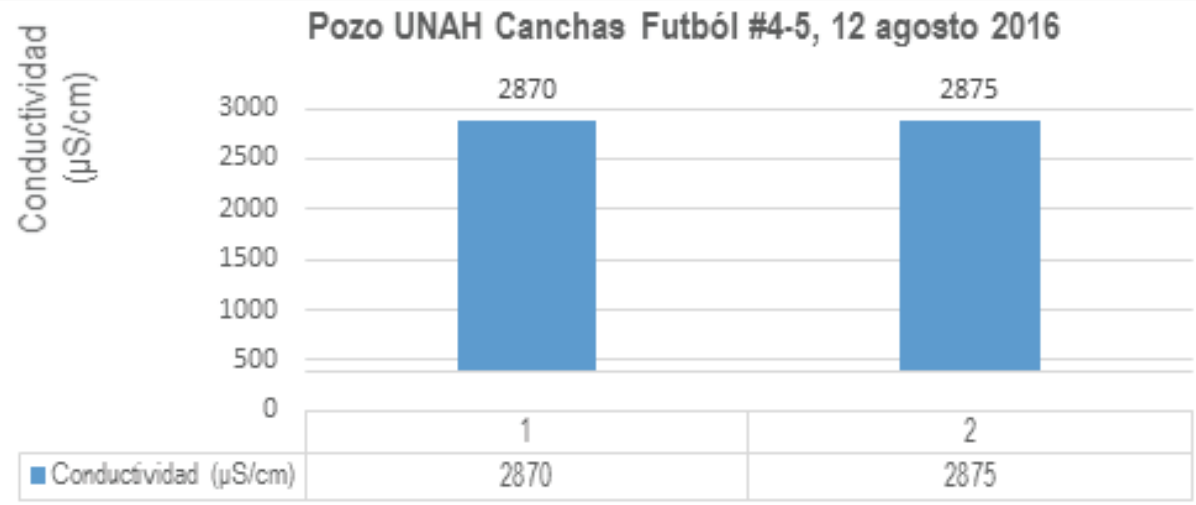

Muestras

Fuente: Elaboración propia.

En el mismo pozo de las canchas de futbol \#4 y \#5 se pueden apreciar también los resultados más altos de las mediciones de dureza del agua en ambas temporadas. Los datos se muestran en el grafico 3 y 4 . (límite de 400 p.p.m.)

\section{Gráfico 3. Dureza total, enero 2016.}

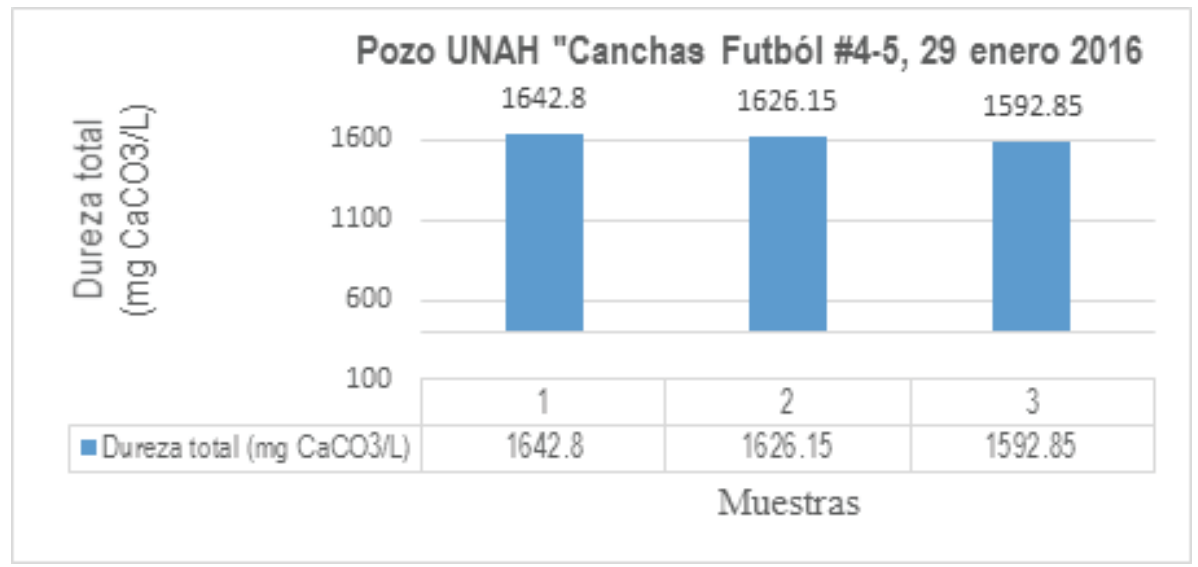

Fuente: Elaboración propia. 
Gráfico 4. Dureza total, agosto 2016.

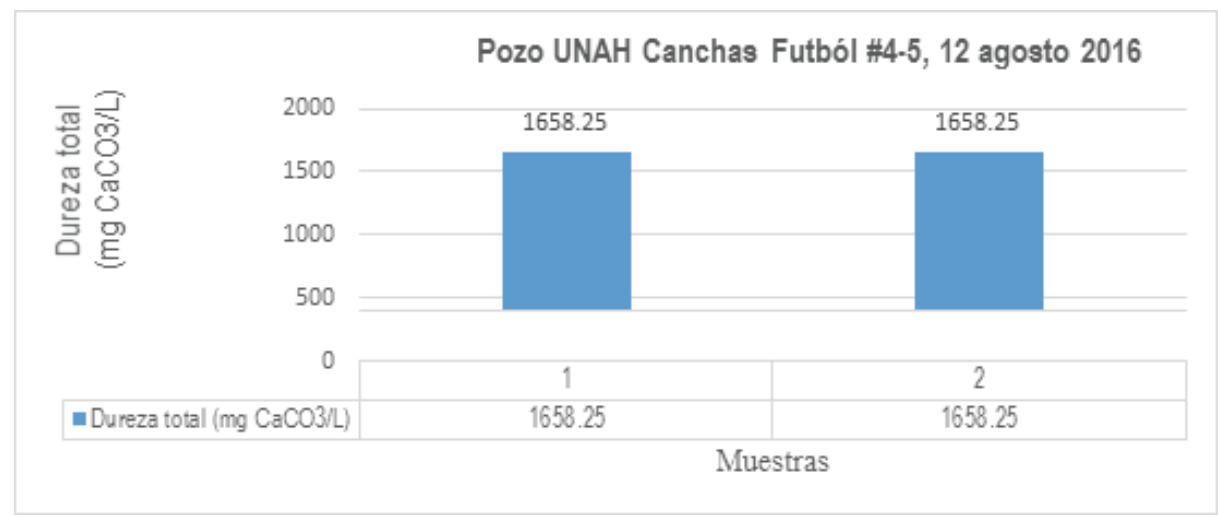

Fuente: Elaboración propia.

Así mismo la presencia de sulfatos fue alta en 4 de los 5 pozos analizados, límite de referencia (250 p.p.m.). Siendo de nuevo el pozo de la cancha 4 y 5 el de mayor contenido de sulfatos y con un marcado incremento en el contenido de sulfatos durante el invierno. Ver gráficos 5-6.

\section{Gráfico 5. Sulfatos en enero 2016.}

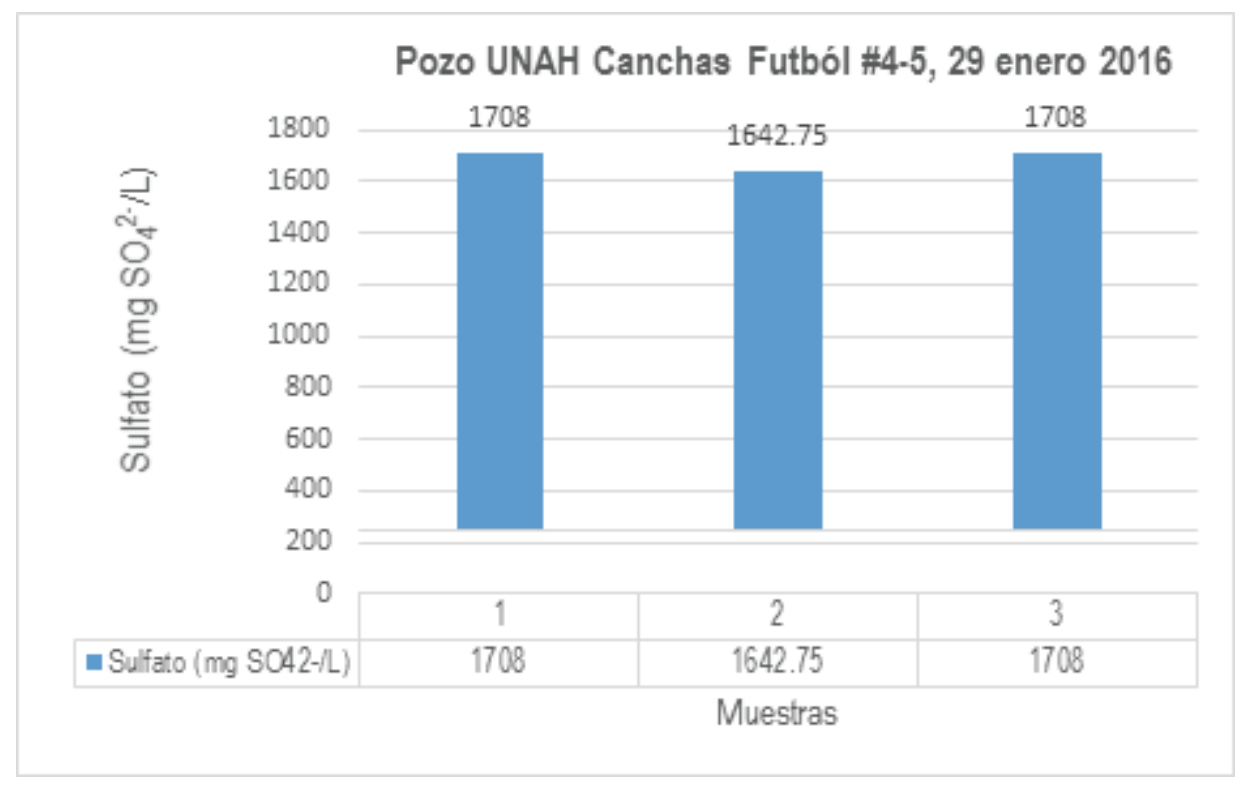

Fuente: Elaboración propia. 
Gráfico 6. Sulfatos en agosto, 2016.

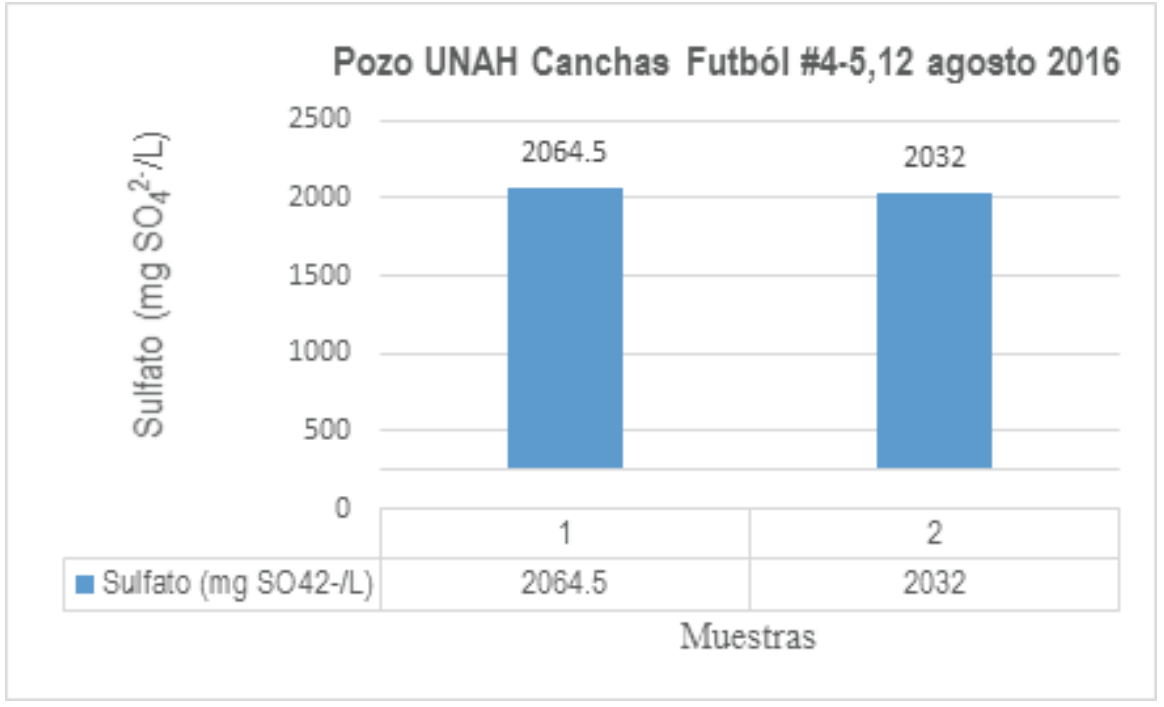

Fuente: Elaboración propia.

El nitrógeno amoniacal fue reportado en una concentración considerablemente alta. Hasta 5 veces más alta del valor de referencia ( 0.5 p.p.m.), como se puede apreciar en ambas temporadas, y con un leve incremento en temporada de lluvia. Siempre este resultado se obtuvo del pozo de las canchas de futbol \#4 y \#5. Ver los gráficos $7-8$.

Gráfico 7. Nitrógeno amoniacal enero, 2016.

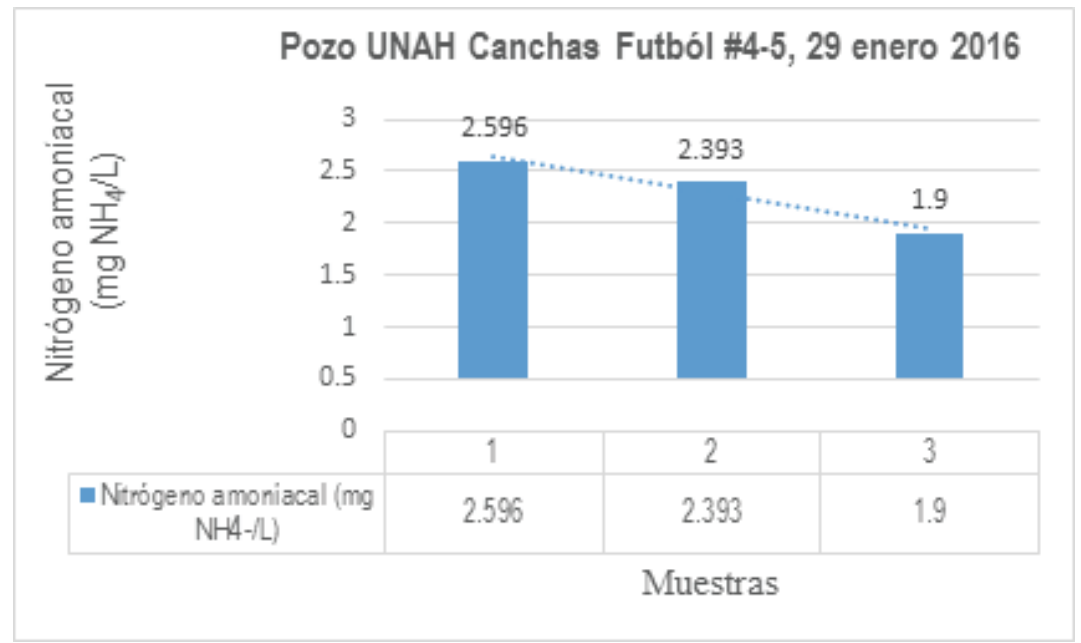

Fuente: Elaboración propia. 
Gráfico 8. Nitrógeno amoniacal agosto, 2016.

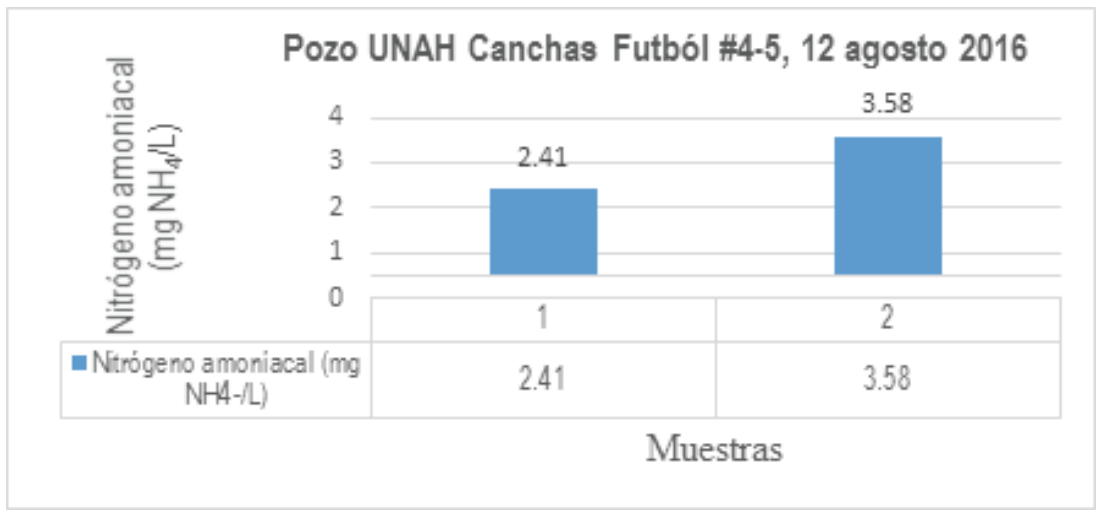

Fuente: Elaboración propia.

Finalmente se encontró solo en una ocasión durante todo el muestreo un valor elevado de hierro. El cual se pudo observar en el pozo ubicado en la zona de carpintería del campus universitario. El límite para el hierro en agua es de 0.3 p.p.m., y el dato encontrado es de 0.57 p.p.m. el cual no esta tan elevado del límite en comparación con los demás parámetros alterados.

\section{DISCUSIÓN Y HALLAZGOS}

Después de analizar los datos obtenidos de cada uno de los pozos del campus se logró identificar al menos cinco parámetros alterados: Dureza total, Conductividad eléctrica, Sulfatos, Hierro y Nitrógeno amoniacal. Todos estos parámetros están clasificados como secundarios según las normas de calidad (Organización Mundial de la Salud, 2006). La presencia de sulfatos elevada, en combinación con la conductividad eléctrica están totalmente relacionadas con la dureza total. Estos son los parámetros que estaban más alterados.

Después de un análisis profundo de los datos analíticos obtenidos, y con la ayuda del Dr. Edward Laws y el Dr. Vincent Wilson, miembros y docentes-investigadores activos del Departamento de Ciencias del Medio Ambiente, Universidad Estatal de Louisiana (LSU), se propusieron las siguientes explicaciones: La elevada cantidad de Sulfatos reportada, estaría relacionada a la presencia de calcio, responsable de la dureza del agua. La forma en que se encontraría este es como yeso, el cual es CaSO4 * $2 \mathrm{H} 2 \mathrm{O}$. Este es un mineral que ocurre naturalmente en muchos procesos geológicas que se 
dan en el entorno de las aguas subterráneas. Así mismo la presencia de yacimientos de sulfato de calcio estaría dando positivo en la detección de hierro, ya que el sulfuro que resulta de la reducción del sulfato es muy afín a metales como el hierro.

La presencia de Nitrógeno Amoniacal podría estar muy relacionada con el uso de fertilizantes en el jardín botánico o al proceso de descomposición de la materia orgánica producto de la vegetación que rodea el área del pozo. La presencia de hierro en el área de carpintería podría deberse a la acumulación en el suelo de desechos de este metal, comúnmente presente en clavos que se emplean en carpintería.

\section{CONCLUSIONES}

La presencia de sulfatos, o de sulfato de calcio si fuere el caso, no representa una amenaza establecida de forma clara a la salud humana. Aunque se ha relacionado el sulfato a reacciones adversas en humanos relacionados con su efecto laxante. (Organización Mundial de la Salud, 2006). Sin embargo, las características organolépticas del agua con alta dureza y presencia de sulfatos hacen que su consumo sea poco atractivo para las personas y en algunos casos un parámetro de calidad para rechazar.

Conforme a los hallazgos del perfil químico de las muestras de agua, el riesgo potencial de emplear el agua subterránea actualmente disponible en ciudad universitaria no es tan alto, ya que los parámetros alterados están definidos como secundarios en la literatura consultada, sin embargo, se ha reportado en la literatura alguna incidencia de efectos laxantes y diarrea en personas expuestas a altas cantidades de sulfato.

El potencial impacto de un perfil químico del agua alterado en los valores de referencia, sobre la comunidad universitaria y visitantes es afortunadamente menos severo; considerando que estos son considerados en la literatura como parámetros secundarios de calidad, que se pueden corregir con medidas de control para remover el calcio.

\section{AGRADECIMIENTOS}

Se agradece ampliamente la colaboración de: Dr. Edward Laws y Dr. Vince Wilson, miembros y docentes-investigadores activos del Departamento de Ciencias del Medio Ambiente, Universidad Estatal de Louisiana (LSU). 


\section{REFERENCIAS BIBLIOGRÁFICAS}

Anna Szynkiewicz, M. R. (2008). Sulfur isotropic study of sulfate in the aquifer of Costa de Hermosillo ( Sonora, Mexico) in realtion to upward intrusion of saline groundwater, irrigation pumping and land cultivation. Applied Geochemistry, volumen (número 23, edición 9), páginas.2539-2558.

Askri, B. (2015). Hydrochemical processes regulating groundwater quality in the coastal plain of Al Musanaah, Sultanate of Oman. Journal of African Earth Sciences, Volumen 106, páginas 87-98.

Christian Herrera, E. C. (2014). Groundwater flow in a relatively old oceanic volcanic island: The Betancuria area, Fuerteventura Island, Canary Islands, Spain. Science of the Total Environment, volumen (número 496), páginas 531-550.

Convención de la Farmacopea de los Estados Unidos de América. (2007). FARMACOPEA DE LOS ESTADOS UNIDOS DE AMERICA. Washington D.C., Estados Unidos de América: THE UNITED STATES PHARMACOPEIAL CONVENTION.

Environmental Protection Agency ( EPA). (2010). water.epa.gov. Retrieved from EPA: http://water.epa.gov/infrastructure/drinkingwater/sourcewater/protection/ upload/2005_05_18_ogwdw_sourcewater_pubs_guide_citguidegwpsp_1990.pdf

M.E. Zabala, M. M. (2015). The origin of groundwater composition in the Pampeano Aquifer underlying the Del Azul Creek basin, Argentina. Science of the Total Environment, Volumen 518/519, páginas 168-188.

Mestres, R. (2013). Quimica Sostenible: Naturaleza, fines y ambito. Mexico D.F., Mexico D.F., Mexico: Editorial Revistas UNAM

Orellana, J. A. (2010). Facultad Regional Rosario, Universidad Tecnologica Nacional. Retrieved from http://www.frro.utn.edu.arl: http://www.frro.utn.edu.ar/repositorio/catedras/civil/ing_sanitaria/Ingenieria_Sanitaria_A4_Capitulo_03_Caracteristicas_del_Agua_ Potable.pdf

Organización Mundial de la Salud. (2006). Guías para la calidad del agua potable (Tercera ed., Vol. 1). Ginebra, Suiza: Ediciones de la OMS, Organización Mundial de la Salud.

Qianlin Zhu, X. L. (2015). Impacts of CO2 leakage into shallow formations on groundwater chemistry. Fuel Processing Technology, volumen (número 135), páginas 162-167.

Secretaría de Recursos Naturales Y Medio Ambiente SERNA. (2005). Geo Honduras. Tegucigalpa, Honduras: @ Programa de las Naciones Unidas para el Medio Ambiente (PNUMAIORPALC).

Universidad Autonoma de Chihuahua Facultad de Ciencias Químicas. (2011). oocities.org. Retrieved from oocities.org: http://www.oocities.org/edrochac/sanitaria/parametros1.pdf 